\section{P09.16 ISOLATION OF NEISSERIA MENINGITIDIS DURING A GONORRHOEA TREATMENT TRIAL}

${ }^{1} \mathrm{SN}$ Taylor*, ${ }^{1} \mathrm{RA}$ Lillis, ${ }^{1} \mathrm{~J}$ Burnett, ${ }^{1} \mathrm{C}$ Cammarata, ${ }^{2} \mathrm{~K}$ Foytich, ${ }^{2} \mathrm{P}$ Dixon, ${ }^{1} \mathrm{~J}$ Siren, ${ }^{1} \mathrm{~T}$ Keller, ${ }^{3} \mathrm{H}$ Tran, ${ }^{3} \mathrm{~S}$ Martin, ${ }^{4} \mathrm{~S}$ Cammarata. 'Louisiana State University Health Sciences Center (LSU); ${ }^{2}$ University of Alabama at Birmingham (UAB); ${ }^{3}$ Louisiana Office of Public Health Laboratory (LA OPH); ${ }^{4}$ Melinta Therapeutics

\section{$10.1136 /$ sextrans-2015-052270.400}

Introduction Neisseria meningitidis (NM) urethritis presenting clinically as Neisseria gonorrhoeae (NG) urethritis and other NG urogenital infections have been described. NM was isolated from participants in a multi-site gonorrhoea treatment trial.

Methods From February 2014 through November 2014, ninetytwo men were enrolled at the LSU site in New Orleans, LA. Urethral and pharyngeal swabs for culture and GenProbe ${ }^{\circledR} \mathrm{CT} / \mathrm{GC}$ were collected from all participants and rectal swabs if receptive anal intercourse was reported. There were 424 culture isolates from the trial, 107 of those from LSU. Preliminary isolate NG identification, confirmatory NG/NM identification and NM serotyping were performed at the LSU, UAB and LA OPH Laboratories, respectively. Urethral NG cultures collected in New Orleans from 2013 were reviewed.

Results Eighty-seven (95\%), 8 (9\%) and $4(4 \%)$ men at the LSU site had positive NG cultures at the urethra, pharynx and rectal sites, respectively. Eight (2\%) isolates from the trial were NM and all were from LSU. Eight (8\%) LSU isolates were NM from $5(5 \%)$ men. Three (3\%) isolates were urethral (Group C-2/ Group B-1), 1 (1\%) was rectal (Group B-1) and 4 (4\%) were pharyngeal isolates (Group B-1/Non-typable-3). One heterosexual and two homosexual men with NM urethritis had purulent discharge and urethral smears identical to gonorrhoea. All Group $B$ isolates were from a vaccinated college student. No NM isolates $(0 / 192)$ were found in urethral cultures collected in New Orleans in 2013.

Conclusion Reports of urogenital NM infections clinically indistinguishable from NG have increased with changes in sexual practices, particularly increase in oral sex. All men with NM urethritis in this report received oral sex. In most clinical settings NM infection will not be recognised because NAAT testing has replaced culture and Gram stains are seldom performed. In addition, sexually transmitted NM infections may also cluster geographically and in time periods.

Disclosure of interest This study was funded by Melinta Therapeutics.

\section{P09.17 RECTAL INFECTION WITH N. GONORRHEAE AND C. TRACHOMATIS IN MEN IN THE UNITED STATES}

${ }^{1} \mathrm{G}$ Tao, ${ }^{2} \mathrm{~K}$ Hoover, ${ }^{3} \mathrm{M}$ Nye, ${ }^{2} \mathrm{P}$ Peters, ${ }^{1} \mathrm{~T}$ Gift, ${ }^{3} \mathrm{~B}$ Body. ${ }^{1} D S T D P$, NCHHSTP, CDC, Atlanta, GA, USA; ${ }^{2}$ DHAP, NCHHSTP, CDC, Atlanta, GA, USA; ${ }^{3}$ Laboratory Corporation of America ${ }^{\circledR}$ Holdings, Burlington, NC, USA

\subsection{6/sextrans-2015-052270.40}

Background US guidelines recommend at least annual rectal chlamydia and gonorrhoea screening in men who have receptive anal intercourse. Data on rectal infection with chlamydia and gonorrhoea in men at the US national level are limited.

Methods In an ongoing collaboration with LabCorp, a large commercial laboratory corporation with a substantial share of the US market, we estimated rectal chlamydia and gonorrhoea positivity in male rectal specimens tested in 2014. Rectal specimens have unique test codes that are consistent among all
LabCorp laboratories. LabCorp had previously validated rectal specimens in collaborative studies.

Results Of 38,411 rectal specimens for chlamydia or gonorrhoea, $96.8 \%(37,170)$ from 28,945 men aged $15-60$ years were tested for both chlamydia and gonorrhoea with nucleic acid amplification tests (NAAT). Of these men, 90.0\% had private insurance and 5.0\% had Medicaid; $15.4 \%$ were aged 15-24 years, $36.4 \%$ 25-34 years, and $22.5 \%$ 35-44 years. Of these 37,170 specimens, $11.0 \%$ were positive for chlamydia, $8.3 \%$ gonorrhoea, and $2.4 \%$ both. Chlamydia and gonorrhoea positivity varied significantly $(\mathrm{p}<0.01)$ by age $(14.9 \%$ and $14.5 \% 15-$ 24 years, $12.2 \%$ and $10.0 \% 25-34$ years, $10.5 \%$ and $6.2 \% 35-$ 44 years, and $7.7 \%$ and $4.2 \%>44$ years, respectively). Of these specimens, $71.6 \%$ were collected by infectious disease doctors, 9.4\% internists, $6.7 \%$ family doctors, and $2.6 \%$ public health providers.

Conclusions This study represents the largest analysis of rectal testing for chlamydial and gonococcal infection in the US Almost all men who receive rectal testing are tested for both chlamydia and gonorrhoea; most rectal testing is performed by infectious disease doctors. NAATs are not FDA cleared for rectal specimens, but many clinical laboratories have validated NAATs under CLIA guidelines and can perform rectal testing. The LabCorp dataset might be the best available source for estimating male rectal infection with chlamydia and gonorrhoea.

\section{P09.18 SUBOPTIMAL PRENATAL TESTING FOR SYPHILIS AND OTHER STDS AMONG COMMERCIALLY-INSURED WOMEN IN THE UNITED STATES, 2013}

G Tao*, R Neblett Fanfair, K Owusu-Edusei, T Gift, KT Bernstein. DSTDP, NCHHSTP, CDC, Atlanta, GA, USA

\subsection{6/sextrans-2015-052270.402}

Background Prenatal syphilis screening is a critical step in the prevention of congenital syphilis infection. US guidelines recommend universal syphilis screening of all pregnant women at the first prenatal visit and again early in the third trimester and at delivery for at risk women.

Methodology We analysed a large commercial claims database to estimate prenatal testing coverage for syphilis among pregnant women aged 15-44 in 2012-2013. We used procedural and diagnostic codes to identify pregnant women with a live birth in 2013. Pregnant women were included if they were enrolled in health plans at least 210 days prior to the date of delivery. Procedural codes were used to identify syphilis, HIV, and other STD testing during prenatal period, defined as 40 weeks before and on the date of delivery.

Results Among 346,811 pregnant women, 82.4\% had syphilis testing before the 28th week of pregnancy or before the third trimester, $84.3 \%$ before the 37 th week of pregnancy, and $85.3 \%$ at any point during the prenatal period. Of these 346,811 women, $71.2 \%$ also had testing for HIV, $83.7 \%$ hepatitis B, $68.0 \%$ chlamydia, and $67.3 \%$ gonorrhoea during the prenatal period. Of 61,112 pregnant women without prenatal syphilis testing before the third trimester, only $16.6 \%$ had any syphilis testing in the third trimester or on the date of delivery. Of 285,699 pregnant women with prenatal syphilis testing before the third trimester, only $22.5 \%$ had any syphilis testing in the third trimester or on the date of delivery.

Conclusions Prenatal screening for syphilis, HIV, and other STDs was suboptimal. Low syphilis testing during the third 